\title{
Public announcements, public assignments and the com- plexity of their logic
}

\author{
Hans van Ditmarsch ${ }^{*}-$ Andreas Herzig $^{* *}-$ Tiago de Lima $^{* * * * * * * *}$ \\ ${ }^{*}$ Department of Logic, University of Sevilla (Spain) \\ http://personal.us.es/hvd \\ ${ }^{* *}$ University of Toulouse, IRIT-CNRS (France) \\ http://www.irit.fr/ Andreas. Herzig \\ ${ }^{* * *}$ University Lille Nord de France (France) \\ ${ }^{* * * *}$ CRIL, UArtois and CNRS (France) \\ http://www.cril.fr/ delima
}

ABSTRACT. We study the extension of public announcement logic PAL by public assignments, that we call PALA. Just as in the case of PAL, the standard procedure for deciding PALA validity, i.e., the use of so-called reduction axioms to translate PALA formulae into formulae in epistemic logic EL, may lead to exponential growth. In this paper, we show that such price is not mandatory, for we provide a polynomial translation of PALA into EL. This is based on abbreviations of subformulae by new propositional letters. Such optimal translation also enables us to show the computational complexity of the problem of deciding PALA validity, which turns out to be coNP-complete in the single-agent case and PSPACE-complete in the multiagent case.

KEYWORDS: knowledge representation and reasoning, dynamic epistemic logic, public announcements, public assignments, automated theorem proving, reduction axioms, complexity.

DOI:10.3166/JANCL.Volume of the issue undefined.1-23 (c) Year of publication undefined Lavoisier, Paris

\section{Introduction}

Dynamic Epistemic Logics (DELs) are extensions of epistemic logic (EL). They provide a logical modeling of actions and events in terms of their effects on the world and on the agents' knowledge. Up to now, research on DELs mainly concentrated on epistemic actions where agents learn that some proposition is true. The archetype of all DELs is Plaza's public announcement logic (PAL) (Plaza, 1989), which has formulae of the form $[! \psi] \varphi$, reading ' $\varphi$ holds after the public announcement of $\psi$ '. However, logics have been proposed where assignments of atomic propositions are added to DELs. They have formulae of the form $[p:=\psi] \varphi$, reading ' $\varphi$ holds after $p$ is assigned the truth value of $\psi$ ' (van Ditmarsch et al., 2005; van Benthem et al., 2006; Kooi, 2007). The semantics of both announcements and assignments is in terms of functions updating Kripke models. We baptise PALA, the extension of PAL with public assignments.

Journal of Applied Non-Classical Logics. Volume Volume of the issue undefined - No. Number of the issue undefined/Year of publication undefined, pages Pages undefined 
2 Journal of Applied Non-Classical Logics. Volume Volume of the issue undefined - No. Number of the issue undefined/Year of publication undefined

PALA was applied in (van Ditmarsch et al., 2007a; van Ditmarsch et al., 2011) to reasoning about actions in Artificial Intelligence.

PALA can be axiomatised by means of so-called reduction axioms. These axioms are equivalences whose iterated application allows to eliminate the dynamic operators from formulae. Such rewrite rules allow therefore to reduce the problem of deciding PALA validity to that of the underlying epistemic logic. Thus, one obtains decision procedures for PALA by reduction to EL and decision procedures for EL.

Clearly, reduction may be suboptimal: basically, as the right hand side of the equivalences may be twice as long as the left hand side, exponential growth of the reduced formula cannot be avoided. Lutz proposed a polynomial reduction from PAL to EL (Lutz, 2006). That transformation makes use of a technique coming from automated theorem proving: in order to avoid exponential growth when putting formulae into conjunctive or disjunctive normal form, subformulae $\chi$ of a given formula $\varphi$ are abbreviated by a new propositional letter $n_{\chi}$. This is done systematically for every subformula of $\varphi$. In the case of modal logics one has to prefix the abbreviation by what is sometimes called a master modality in order to guarantee that the equivalence holds not only in the actual world, but throughout the model (that can be thought of as being point-generated). The transformation preserves validity, and the length of the resulting formula is polynomial in the length of the original formula $\varphi$.

In this paper we pursue the quest of polynomial reduction procedures. We show that Lutz's abbreviation technique can be adapted to PALA. This leads us to an optimal method for deciding PALA validity. It follows that the problem of deciding validity is coNP-complete for single-agent PALA, and PSPACEcomplete for multiagent PALA.

The remainder of this paper is organized as follows: Section 2 introduces public announcement logic with assignment PALA. Section 3 contains the standard reduction from PALA to PAL, which is nonoptimal. Section 4 recalls Lutz's optimal reduction from PAL to EL, and Section 5 provides an optimal reduction from PALA to EL. Section 6 addresses multiagent PALA. Section 7 concludes. ${ }^{1}$

\section{Public announcement logic with assignment}

In this section we recall public announcement logic with assignment (PALA). We only present the single-agent case, of which the multiagent case is a straightforward extension.

\subsection{Syntax}

The language of public announcement logic with assignment PALA is the set of formulae $\varphi$ and assignments $\sigma$ that is defined by the following BNF:

$$
\begin{aligned}
& \varphi::=p|\neg \varphi|(\varphi \wedge \varphi)|\mathbf{K} \varphi|[! \varphi] \varphi \mid[\sigma] \varphi \\
& \sigma::=\epsilon \mid p:=\varphi, \sigma
\end{aligned}
$$

where $p$ ranges over the countable set of propositional letters $P$ and $\epsilon$ is the empty assignment. We write $\left[p_{1}:=\psi_{1}, \ldots, p_{k}:=\psi_{k}\right] \varphi$ instead of $\left[p_{1}:=\psi_{1}, \ldots, p_{k}:=\psi_{k}, \epsilon\right] \varphi$.

Let $\alpha$ be one of ! $\varphi$ or $\sigma$; the formula $[\alpha] \varphi$ reads ' $\varphi$ holds after all possible executions of $\alpha$ '. The event $! \varphi$ is the public announcement of $\varphi$; and the event $p:=\varphi$ is the public assignment of $\varphi$ to the atom $p$. For

1. This paper extends and improves the second part of (van Ditmarsch et al., 2007a). The first part of that paper has been extended and improved in (van Ditmarsch et al., 2011). 
example, $p:=(q \wedge \neg q)$ is an assignment making $p$ false, and $\mathbf{K}[p:=(q \wedge \neg q)] \neg p$ is a formula expressing that the agent knows this.

Every $[\sigma]$ is an assignment operator, and every $[! \psi]$ is an announcement operator. A dynamic operator is either an assignment operator or an announcement operator. The language of public announcement logic (PAL) is the subset of the language of PALA where no assignment operators occur, the language of epistemic logic with assignment (ELA) is the subset of the language of PALA where no announcement operators occur, and the language of epistemic logic EL is the subset of the language of ELA where no dynamic operators occur.

We use the standard abbreviations for $\perp, \top, \vee, \rightarrow$, and $\leftrightarrow: \perp$ is $p \wedge \neg p$ (for some propositional letter $p)$, $\top$ is $\neg \perp, \varphi \vee \psi$ is $\neg(\neg \varphi \wedge \neg \psi), \varphi \rightarrow \psi$ is $\neg(\varphi \wedge \neg \psi)$, and $\varphi \leftrightarrow \psi$ is $(\varphi \rightarrow \psi) \wedge(\psi \rightarrow \varphi)$.

The language just defined allows for complex assignments where a propositional letter appears more than once on the left hand side of the operator ' $:=$ '. Our semantics will make that in such cases only the leftmost occurrence of this propositional letter matters.

Every assignment $\sigma$ may be considered as a mapping from $P$ to formulae of the language of PALA. This is recursively defined as follows:

$$
\begin{gathered}
(q) \epsilon=q \\
(q)(p:=\varphi, \sigma)= \begin{cases}\varphi & \text { if } q=p \\
(q) \sigma & \text { otherwise }\end{cases}
\end{gathered}
$$

For example, $(p) \epsilon=p,(p)(p:=\neg p)=\neg p$, and $(p)(p:=q, q:=p, p:=r)=q$. Then, without loss of generality, an assignment $\sigma$ can be seen as a finite, and hence partial, function from propositional letters to formulae. The domain of $\sigma$ can be defined recursively as follows:

$$
\begin{aligned}
\operatorname{dom}(\epsilon) & =\emptyset \\
\operatorname{dom}(p:=\varphi, \sigma) & =\{p\} \cup \operatorname{dom}(\sigma)
\end{aligned}
$$

It will be sometimes convenient to use finite sets $\left\{p_{1}:=\varphi_{1}, \ldots, p_{n}:=\varphi_{n}\right\}$ to denote assignments; the empty assignment $\epsilon$ is then identified with $\emptyset$.

The function len returns the length of a given expression, where an expression is a formula or an assignment. It basically counts the number of symbols to write down the given expression (without parentheses). ${ }^{2}$ In other words:

$$
\begin{aligned}
\operatorname{len}(p) & =1 \\
\operatorname{len}(\neg \varphi) & =1+\operatorname{len}(\varphi) \\
\operatorname{len}(\varphi \wedge \psi) & =1+\operatorname{len}(\varphi)+\operatorname{len}(\psi) \\
\operatorname{len}(\mathbf{K} \varphi) & =1+\operatorname{len}(\varphi) \\
\operatorname{len}([! \psi] \varphi) & =1+\operatorname{len}(\psi)+\operatorname{len}(\varphi) \\
\operatorname{len}([\sigma] \varphi) & =1+\operatorname{len}(\sigma)+\operatorname{len}(\varphi) \\
\operatorname{len}(\sigma) & =\sum_{p \in \operatorname{dom}(\sigma)} \operatorname{len}((p) \sigma)
\end{aligned}
$$

2. Strictly speaking, the propositional letter $p$ has to be encoded as a binary number $n$, and the length of $p$ is therefore $\log _{2} n$. It follows that the number of symbols required to write down a formula $\varphi$ is $\operatorname{len}(\varphi) \times \log _{2}(\operatorname{len}(\varphi)+1)$. This, however, does not change our results. In particular, the reduction of Proposition 12 remains polynomial. 
4 Journal of Applied Non-Classical Logics. Volume Volume of the issue undefined - No. Number of the issue undefined/Year of publication undefined

For example, len $(\perp)=\operatorname{len}(p \wedge \neg p)=4$, len $(\top)=\operatorname{len}(\neg \perp)=5$, and

$$
\begin{aligned}
\operatorname{len}(\varphi \leftrightarrow \psi) & =\operatorname{len}(\neg(\varphi \wedge \neg \psi) \wedge \neg(\psi \wedge \neg \varphi)) \\
& =1+\operatorname{len}(\neg(\varphi \wedge \neg \psi))+\operatorname{len}(\neg(\psi \wedge \neg \varphi)) \\
& =1+(\operatorname{len}(\varphi)+\operatorname{len}(\psi)+3)+(\operatorname{len}(\varphi)+\operatorname{len}(\psi)+3) \\
& =7+(2 \times \operatorname{len}(\varphi))+(2 \times \operatorname{len}(\psi)) ;
\end{aligned}
$$

And the length of $[p:=q, q:=p \wedge q, \epsilon] \mathbf{K} p$ is $1+\operatorname{len}(p:=q, q:=p \wedge q, \epsilon)+\operatorname{len}(\mathbf{K} p)=1+(1+3+0)+2=7$.

\subsection{Semantics}

Formulae of the language of PALA are interpreted in pointed models of epistemic logic.

First, a model of epistemic logic (EL model) is a tuple $M=\langle W, R, V\rangle$ such that:

- $W$ is a non-empty set of possible worlds,

$-R \subseteq W \times W$ is an equivalence relation, and

- $V: P \rightarrow \wp(W)$ associates an interpretation $V(p) \subseteq W$ to each $p \in P$.

For every $w \in W$, the pair $(M, w)$ is a pointed $\mathrm{EL}$ model.

For convenience, we define $R(w)=\{u \mid(w, u) \in R\}$. The elements of $R(w)$ are the worlds the agent considers possible at $w$.

The satisfaction relation $\models$ between pointed EL models $(M, w)=(\langle W, R, V\rangle, w)$ and PALA formulae is inductively defined as follows:

$$
\begin{aligned}
& M, w=p \quad \text { iff } \quad w \in V(p) \\
& M, w \vDash \neg \varphi \quad \text { iff } \quad M, w \not \varphi \\
& M, w \vDash \varphi \wedge \psi \quad \text { iff } \quad M, w \vDash \varphi \text { and } M, w \vDash \psi \\
& M, w \vDash \mathbf{K} \varphi \quad \text { iff } \quad R(w) \subseteq \llbracket \varphi \rrbracket_{M} \\
& M, w \vDash[! \varphi] \psi \quad \text { iff } \quad M, w \vDash \varphi \text { implies } M^{! \varphi}, w \vDash \psi \\
& M, w \vDash[\sigma] \varphi \quad \text { iff } \quad M^{\sigma}, w \vDash \varphi
\end{aligned}
$$

where $\llbracket \varphi \rrbracket_{M}=\{w \mid M, w \models \varphi\}$ is the extension of $\varphi$ in $M$, and where the models $M^{! \varphi}$ and $M^{\sigma}$ are updates of the epistemic model $M$, that are respectively defined as:

$$
\begin{aligned}
M^{! \varphi} & =\left\langle W^{! \varphi}, R^{! \varphi}, V^{! \varphi}\right\rangle & M^{\sigma} & =\left\langle W^{\sigma}, R^{\sigma}, V^{\sigma}\right\rangle \\
W^{! \varphi} & =W & W^{\sigma} & =W \\
R^{! \varphi} & =R \cap\left(\llbracket \varphi \rrbracket_{M} \times \llbracket \varphi \rrbracket_{M}\right) & R^{\sigma} & =R \\
V^{! \varphi} & =V & V^{\sigma}(p) & =\llbracket(p) \sigma \rrbracket_{M}
\end{aligned}
$$

To illustrate the semantics of PALA, let $(M, w)$ be any pointed EL model. We have $M, w \vDash[p:=\perp] \neg p$ because $V^{p:=\perp}(p)=\llbracket(p)(p:=\perp) \rrbracket_{M}=\llbracket \perp \rrbracket_{M}=\emptyset$; and we have $M, w \vDash[! p] \mathbf{K} p$ because $R^{! p}(w) \subseteq \llbracket p \rrbracket_{M}$.

Note that if $(M, w)$ is a pointed EL model then $M^{p:=\varphi}$ is an EL model; and if $M, w \vDash \varphi$ (which is the relevant case in the truth condition) then $M^{! \varphi}$ is an EL model. 
REMARK 1. - Our definition of updates by announcements is a well-known variation of the standard definition where $W^{! \varphi}=\llbracket \varphi \rrbracket_{M}$ and $V^{! \varphi}(p)=V(p) \cap \llbracket \varphi \rrbracket_{M}$; see e.g. (Kooi, 2007).

A PALA formula $\varphi$ is valid in a model $M=\langle W, R, V\rangle$, noted $M \vDash \varphi$, if and only if $\llbracket \varphi \rrbracket_{M}=W$; A formula $\varphi$ is PALA valid, noted $\models_{\text {PALA }} \varphi$, if and only if for all pointed EL models $(M, w),(M, w) \vDash \varphi$; and $\varphi$ is PALA satisfiable if and only if $\nvdash_{\text {PALA }} \neg \varphi$.

For example, $[p:=\perp] \neg p$ and $[! p] \mathbf{K} p$ are PALA valid, for atomic $p$. Another example is $\vDash_{\text {PALA }}[p:=q, q:=p \wedge r](p \wedge \neg q \wedge s) \leftrightarrow(q \wedge \neg(p \wedge r) \wedge s)$.

This by the way also illustrates that the elements of a complex assignment can be thought as being executed in parallel. Just as in PAL, $[! \varphi] \varphi$ is not always PALA valid, nor is the stronger $[! \varphi] \mathbf{K} \varphi$.

The corresponding semantic notions of validity and satisfiability are defined likewise for PAL, ELA and EL.

Proposition 2. - Both PALA and ELA are conservative extensions of EL: if $\varphi$ is an EL formula then both $\models_{\mathrm{PALA}} \varphi$ iff $\models_{\mathrm{EL}} \varphi$, and $\models_{\mathrm{ELA}} \varphi$ iff $\models_{\mathrm{EL}} \varphi$.

\section{Suboptimal reduction}

In this section we present the method that is common in dynamic epistemic logics to prove decidability, viz. by means of reduction axioms.

\subsection{Reduction axioms}

Logic EL is the well-known logic S5, whose axiomatization consists of CPL (the tautologies of propositional classical logic), rules RM (Modus Ponens) and RN (Necessitation), and axiom schemes K, T and 5.

$\begin{array}{ll}\text { from } \varphi \text { and } \varphi \rightarrow \psi \text { infer } \psi & \mathrm{RM} \\ \text { from } \varphi \text { infer } \mathbf{K} \varphi & \mathrm{RN} \\ \mathbf{K}(\varphi \rightarrow \psi) \rightarrow(\mathbf{K}(\varphi \rightarrow \mathbf{K} \psi) & \mathrm{K} \\ \mathbf{K} \varphi \rightarrow \varphi & \mathrm{T} \\ \neg \mathbf{K} \varphi \rightarrow \mathbf{K} \neg \varphi & 5\end{array}$

The axiomatization of PALA extends that of EL by so-called reduction axioms: equivalences for all possible combinations of assignments with the logical connectives. 
6 Journal of Applied Non-Classical Logics. Volume Volume of the issue undefined - No. Number of the issue undefined/Year of publication undefined

Proposition 3 ((van Ditmarsch et al., 2005; van Benthem et Al., 2006; van Ditmarsch et al., 2007в)). The following formula schemes are PALA valid.

$\begin{array}{rlrl}{[\sigma] p} & \leftrightarrow(p) \sigma, \text { for } p \in P & & \operatorname{Red}_{:=, P} \\ {[! \psi] p} & \leftrightarrow(\psi \rightarrow p), \text { for } p \in P & \operatorname{Red}_{!, P} \\ {[\sigma] \neg \varphi} & \leftrightarrow \neg[\sigma] \varphi & \operatorname{Red}_{:=,\urcorner} \\ {[! \psi] \neg \varphi} & \leftrightarrow(\psi \rightarrow \neg[! \psi] \varphi) & \operatorname{Red}_{!, \neg} \\ {[\sigma]\left(\varphi_{1} \wedge \varphi_{2}\right)} & \leftrightarrow\left([\sigma] \varphi_{1} \wedge[\sigma] \varphi_{2}\right) & & \operatorname{Red}_{:=, \wedge} \\ {[! \psi]\left(\varphi_{1} \wedge \varphi_{2}\right)} & \leftrightarrow\left([! \psi] \varphi_{1} \wedge[! \psi] \varphi_{2}\right) & \operatorname{Red}_{!, \wedge} \\ {[\sigma] \mathbf{K} \varphi} & \leftrightarrow \mathbf{K}[\sigma] \varphi & \operatorname{Red}_{:=, \mathbf{K}} \\ {[! \psi] \mathbf{K} \varphi} & \leftrightarrow(\psi \rightarrow \mathbf{K}[! \psi] \varphi) & \operatorname{Red}_{!, \mathbf{K}}\end{array}$

The right hand side of the above equivalences is simpler than their left hand side, in the sense that the dynamic operator is either eliminated (in the case of the first two equivalences) or 'pushed inward' (in the case of the other equivalences); see e.g. (Kooi, 2007) for a precise definition of what it means to be 'simpler'. Such equivalences are called reduction axioms. To apply them means to replace subformulae of a given formula that match the left hand side of some reduction axiom, by its right hand side. When we do that we apply the rule of replacement of equivalents RRE. The latter preserves validity because the below inference rules do so.

Proposition 4. - The following inference rules preserve PALA validity.

$$
\begin{array}{ll}
\text { from } \varphi \leftrightarrow \varphi^{\prime} \text { infer }[! \psi] \varphi \leftrightarrow[! \psi] \varphi^{\prime} & \mathrm{RE}_{!}^{r} \\
\text { from } \psi \leftrightarrow \psi^{\prime} \text { infer }[! \psi] \varphi \leftrightarrow\left[! \psi^{\prime}\right] \varphi & \mathrm{RE}_{!}^{l} \\
\text { from } \varphi \leftrightarrow \varphi^{\prime} \text { infer }[\sigma] \varphi \leftrightarrow[\sigma] \varphi^{\prime} & \mathrm{RE}_{:=}^{r} \\
\text { from } \psi \leftrightarrow \psi^{\prime} \text { infer }[p:=\psi, \sigma] \varphi \leftrightarrow\left[p:=\psi^{\prime}, \sigma\right] \varphi & \mathrm{RE}_{:=}^{l}
\end{array}
$$

Proof. - $\mathrm{RE}_{!}^{r}$ and $\mathrm{RE}_{!}^{l}$ follow from the proof in (van Ditmarsch et al., 2007b) that the rule of replacement of equivalents preserves PAL validity.

For $\mathrm{RE}_{:=}^{r}$, suppose $\llbracket \varphi \rrbracket_{M}=\llbracket \varphi^{\prime} \rrbracket_{M}$ for every model $M$, and let $M$ be some model. Then $\llbracket[\sigma] \varphi \rrbracket_{M}=$ $\llbracket \varphi \rrbracket_{M^{\sigma}}$. By hypothesis the latter equals $\llbracket \varphi^{\prime} \rrbracket_{M^{\sigma}}$, which in turn is equal to $\llbracket[\sigma] \varphi^{\prime} \rrbracket_{M}$.

For $\mathrm{RE}_{:=}^{l}$, suppose $\llbracket \psi \rrbracket_{M}=\llbracket \psi^{\prime} \rrbracket_{M}$ for every model $M$, and let $M$ be some model. Then $M^{p:=\psi, \sigma}=$ $M^{p:=\psi^{\prime}, \sigma}$.

Proposition 3 provides reduction axioms for all combinations of dynamic operators with EL connectives. Under the condition that we start with some dynamic operator $[! \psi]$ that is innermost (in the sense that it has no other dynamic operator in its scope) we have reduction axioms for all cases, allowing to eliminate $[! \psi]$ : the resulting formula has one dynamic operator less than the original formula. 
Remark 5. - We did not state reduction axioms for combinations of dynamic operators with dynamic operators. Such axioms exist for PAL and ELA, viz. $[! \psi][! \chi] \varphi \leftrightarrow[!(\psi \wedge[! \psi] \chi)] \varphi$ and $\left[\sigma_{1}\right]\left[\sigma_{2}\right] \varphi \leftrightarrow$ $\left[\sigma_{1} \circ \sigma_{2}\right] \varphi$, where $\sigma_{1} \circ \sigma_{2}$ is the composition of $\sigma_{1}$ and $\sigma_{2}$ that is defined as function composition. However, there can be no reduction axiom for $[\sigma][! \psi] \varphi$. In particular the schema $[\sigma][! \psi] \varphi \leftrightarrow[!([\sigma] \psi)][\sigma] \varphi$ is PALA invalid. To see this replace $\sigma$ by $p:=p \wedge \neg \mathbf{K} p$ and replace both $\psi$ and $\varphi$ by $p$. Then

$$
\begin{aligned}
{[p:=p \wedge \neg \mathbf{K} p][! p] p } & \leftrightarrow[p:=p \wedge \neg \mathbf{K} p] \top \\
& \leftrightarrow \top \\
{[!([p:=p \wedge \neg \mathbf{K} p] p)][p:=p \wedge \neg \mathbf{K} p] p } & \leftrightarrow[!([p:=p \wedge \neg \mathbf{K} p] p)](p \wedge \neg \mathbf{K} p) \\
& \leftrightarrow[!(p \wedge \neg \mathbf{K} p)](p \wedge \neg \mathbf{K} p) \\
& \leftrightarrow \neg(p \wedge \neg \mathbf{K} p)
\end{aligned}
$$

So the former formula is PALA valid because it reduces to $T$, while the latter is not because the last line is not valid in S5.

The absence of such reduction principles does not hurt. For our purposes we do not need reduction axioms for sequences of dynamic (announcement or assignment) operators, because by iterating the elimination of an innermost dynamic operator we end up with a formula having no dynamic operator at all; in other words: an EL formula. Call $\operatorname{red}(\varphi)$ the result of rewriting $\varphi$ by the above reduction axioms until all dynamic operators are eliminated.

Theorem 6. - Let $\varphi$ be a PALA formula. Then:

1) $\operatorname{red}(\varphi)$ is an EL formula

2) $\models_{\text {PALA }} \varphi \leftrightarrow \operatorname{red}(\varphi)$

3) $\models_{\mathrm{PALA}} \varphi$ if and only if $\models_{\mathrm{EL}} \operatorname{red}(\varphi)$

Proof. - This is proved just as for the other dynamic epistemic logics having reduction axioms for all logical operators of EL, see e.g. (van Ditmarsch et al., 2007b; Kooi, 2007).

For the first item, we use that the right hand sides of the reduction axioms are simpler than their left hand sides in the sense that the dynamic operator is either eliminated or pushed inwards: as the function red is applied until there is no more dynamic operator, the result has no dynamic operator any more.

The proof of the second item uses that red applies valid equivalences (Proposition 3) and that the inference rule RRE preserves PALA validity (due to Proposition 4).

The third item follows from the first two and Proposition 2.

The last item of Theorem 6 tells us that PALA validity of $\varphi$ can be checked by applying some EL decision procedure to $\operatorname{red}(\varphi)$. While the problem of deciding EL validity is in coNP, this does not entitle us to claim the same for the problem of deciding validity in PALA, as we are going to see now.

\subsection{Reduction may lead to exponential growth}

We have just seen that red provides a decision procedure for PALA validity. However, $\operatorname{red}(\varphi)$ may be exponentially longer than $\varphi$, 
8 Journal of Applied Non-Classical Logics. Volume Volume of the issue undefined - No. Number of the issue undefined/Year of publication undefined

ExAmple 7. - Consider the family of formulae $\psi_{n}$ that is inductively defined by:

$$
\begin{aligned}
\psi_{0} & =p_{0} \\
\psi_{n+1} & =\left[p_{n}:=p_{n+1} \wedge p_{n+1}\right] \psi_{n}
\end{aligned}
$$

Successively applying the reduction axioms to the innermost assignment we get:

$$
\begin{aligned}
{\left[p_{n-1}:=p_{n} \wedge p_{n}\right] \ldots\left[p_{1}:=p_{2} \wedge p_{2}\right]\left[p_{0}:=p_{1} \wedge p_{1}\right] p_{0} } & \leftrightarrow\left[p_{n-1}:=p_{n} \wedge p_{n}\right] \ldots\left[p_{1}:=p_{2} \wedge p_{2}\right]\left(p_{1} \wedge p_{1}\right) \\
& \leftrightarrow\left[p_{n-1}:=p_{n} \wedge p_{n}\right] \ldots\left(\left(p_{2} \wedge p_{2}\right) \wedge\left(p_{2} \wedge p_{2}\right)\right) \\
& \vdots \\
& \left.\leftrightarrow\left(\ldots\left(p_{n} \wedge p_{n}\right) \wedge \ldots\right) \ldots\right)
\end{aligned}
$$

The last formula cannot be reduced any more, and it contains $2^{n}$ occurrences of the propositional letter $p_{n}$.

One may hope to find a polynomial reduction which, given a PALA formula $\varphi$, produces an EL formula $\varphi^{\prime}$ such that $\varphi \leftrightarrow \varphi^{\prime}$ is PALA valid. However, this cannot be the case. Lutz showed that, if the underlying epistemic logic is $\mathrm{K}$, then there is a family of PAL formulae $\varphi_{n}$ such that for every $\varphi_{n}$, any equivalent EL formula is exponentially longer than $\varphi_{n}$ (Lutz, 2006, Theorem 2). While he only conjectured that his result transfers to S5, French et al. recently provided a proof (French et al., 2011).

Notwithstanding, there may still be a polynomial transformation preserving satisfiability equivalence (which is a weaker requirement than logical equivalence). The aim of the rest of the paper is to provide such a transformation.

\section{Optimal reduction for PAL}

For PAL, Lutz proposed a polynomial reduction to EL preserving satisfiability (Lutz, 2006). His transformation adapts a technique originating from automated theorem proving. We present this transformation now.

\subsection{The abbreviation technique for propositional logic}

When putting formulae of classical propositional logic into conjunctive or disjunctive normal form one faces the problem of exponential growth. For example, the straightforward application of the law of distributivity to $(\varphi \wedge \psi) \vee \chi$ leads to $(\varphi \vee \chi) \wedge(\psi \vee \chi)$ : the subformula $\chi$ occurs twice in the resulting formula, and iteration of the distribution may produce formulae that are exponentially longer than the original formula.

In automated theorem proving, a standard technique to obtain polynomial normal forms is to replace complex subformulae $\chi$ of a given formula $\varphi$ by a new propositional letter $n_{\chi}$ and to conjoin the resulting formula and the equivalence $n_{\chi} \leftrightarrow \chi$; see e.g. (Nonnengart \& Weidenbach, 2001). For example, the complex subformula $\chi$ in $(\varphi \wedge \psi) \vee \chi$ is replaced by a new atomic formula $n_{\chi}$, resulting in $(\varphi \wedge \psi) \vee n_{\chi}$, to which the law of distributivity can be applied without leading to exponential growth. This transformation preserves satisfiability, in the sense that $(\varphi \wedge \psi) \vee \chi$ is satisfiability equivalent to $\left((\varphi \wedge \psi) \vee n_{\chi}\right) \wedge\left(n_{\chi} \leftrightarrow \chi\right)$. 
The abbreviation $n_{\chi} \leftrightarrow \chi$ must also be put in normal form, hence subformulae have to be abbreviated systematically. This is done by associating to $\varphi$ the following set of bi-implications (where $\operatorname{SF}(\varphi)$ is the set of subformulae of $\varphi$ ):

$$
\begin{aligned}
B_{\varphi}= & \left\{n_{p} \leftrightarrow p \mid p \in \operatorname{SF}(\varphi) \cap P\right\} \cup \\
& \left\{n_{\neg \psi} \leftrightarrow \neg n_{\psi} \mid \neg \psi \in \operatorname{SF}(\varphi)\right\} \cup \\
& \left\{n_{\psi_{1} \wedge \psi_{2}} \leftrightarrow n_{\psi_{1}} \wedge n_{\psi_{2}} \mid \psi_{1} \wedge \psi_{2} \in \operatorname{SF}(\varphi)\right\}
\end{aligned}
$$

This transformation preserves satisfiability: the original formula $\varphi$ is satisfiable in classical propositional logic if and only if $n_{\varphi} \wedge\left(\wedge B_{\varphi}\right)$ is satisfiable in classical propositional logic. Moreover the length of the resulting formula is polynomial in the length of the original formula $\varphi$.

\subsection{The abbreviation technique for epistemic logic}

In the case of epistemic logic one has to take into account the modal operator of knowledge: The definition of $B_{\varphi}$ is augmented by the set

$$
\left\{n_{\mathbf{K} \psi} \leftrightarrow \mathbf{K} n_{\psi} \mid \mathbf{K} \psi \in \mathrm{SF}(\varphi)\right\}
$$

Moreover, the abbreviations in $B_{\varphi}$ have to be prefixed by what is sometimes called a master modality. This guarantees that the equivalences hold not only in the actual world, but throughout the model (that is thought of as being point-generated). Then the original formula $\varphi$ is EL satisfiable if and only if $n_{\varphi} \wedge \mathbf{K}\left(\wedge B_{\varphi}\right)$ is EL satisfiable.

\subsection{Lutz's optimal reduction for $\mathrm{PAL}$}

The abbreviation method does not extend straightforwardly to PAL. To see this suppose we again augment the definition of $B_{\varphi}$ by the set

$$
\left\{n_{[! \varphi] \psi} \leftrightarrow\left[! n_{\varphi}\right] n_{\psi} \mid[! \varphi] \psi \in \mathrm{SF}(\varphi)\right\}
$$

Consider the PAL unsatisfiable formula $\varphi=\neg[! p] \mathbf{K} p$. (Unsatisfiability is the case because $[! p] \mathbf{K} p$ reduces to $p \rightarrow \mathbf{K}(p \rightarrow p)$, which is EL valid.) However, the formula

$$
\begin{aligned}
n_{\neg[! p] \mathbf{K}_{p}} \wedge \mathbf{K}( & \left(n_{\neg[! p] \mathbf{K} p} \leftrightarrow \neg n_{[! p] \mathbf{K} p}\right) \wedge \\
& \left(n_{[! p] \mathbf{K} p} \leftrightarrow\left[! n_{p}\right] n_{\mathbf{K} p}\right) \wedge \\
& \left(n_{\mathbf{K} p} \leftrightarrow \mathbf{K} n_{p}\right) \wedge \\
& \left.\left(n_{p} \leftrightarrow p\right)\right)
\end{aligned}
$$

is PAL satisfiable in the pointed model $(\langle W, R, V\rangle, w)$, where $W=\{w, v\}, R=W \times W$, and $V(p)=V\left(n_{p}\right)=$ $V\left(n_{\neg[! p] \mathbf{K} p}\right)=\{w\}$, and $V\left(n_{\mathbf{K} p}\right)=\emptyset$, and $V\left(n_{[! p] \mathbf{K} p}\right)=\{v\}$. In particular note that $(\langle W, R, V\rangle, w) \not \forall\left[! n_{p}\right] n_{\mathbf{K} p}$, telling us that this naive extension does not allow to correctly abbreviate subformulae that are in the scope of an announcement.

Lutz succeeded in finding a polynomial transformation $\operatorname{red}_{\mathrm{PAL}}$ mapping PAL formulae to EL formulae that preserves satisfiability equivalence: for every $\operatorname{PAL}$ formula $\varphi, \varphi$ is PAL satisfiable iff $\operatorname{red}_{\mathrm{PAL}}(\varphi)$ is EL satisfiable (Lutz, 2006, Lemma 7), and the length of the reduction len $\left(\operatorname{red}_{\mathrm{PAL}}(\varphi)\right)$ is quadratic in $\operatorname{len}(\varphi)$ (Lutz, 2006, Lemma 6). His trick is to encode the modal context of a sub-formula as a superscript of the new propositional letter. We do not give the definition of red $d_{\text {PAL }}$ here: it is a particular case of our polynomial transformation from PALA to EL. 
10 Journal of Applied Non-Classical Logics. Volume Volume of the issue undefined - No. Number of the issue undefined/Year of publication undefined

\section{Optimal reduction for ELA and PALA}

In the rest of the paper we extend Lutz's abbreviation technique to PALA. For the sake of clarity, we split the exposition into two parts. In the first part, we address the fragment of PALA called ELA (i.e., with assignments but without announcements), and in the second, we address the entire logic PALA.

\subsection{From ELA to EL}

Call a context a list $\lambda=\left(\lambda_{1}, \ldots, \lambda_{m}\right)$ of assignments, where $m \geq 0$. The empty context is noted (), and the concatenation of $\lambda$ and the assignment $\sigma$ is $\lambda \cdot \sigma=\left(\lambda_{1}, \ldots, \lambda_{m}, \sigma\right)$. The $k$-th element of $\lambda$ is noted $\lambda_{k}$.

The assignments governing a subformula of a given formula $\varphi$ make up its context in $\varphi$ : for every subformula $\chi$ of $\varphi$, the context of $\chi$ in $\varphi$ is the sequence of assignment operators governing $\chi$ in $\varphi$.

Definition 8. - Given a context $\lambda$ and an input formula $\varphi$ we recursively define the set $\operatorname{CS}(\lambda, \varphi)$ of contextualised subformulae of $\varphi$ given $\lambda$ :

$$
\begin{aligned}
C S(\lambda, p) & =\{\langle\lambda, p\rangle\} \\
C S(\lambda, \neg \chi) & =C S(\lambda, \chi) \cup\{\langle\lambda, \neg \chi\rangle\} \\
C S\left(\lambda, \chi_{1} \wedge \chi_{2}\right) & =C S\left(\lambda, \chi_{1}\right) \cup C S\left(\lambda, \chi_{2}\right) \cup\left\{\left\langle\lambda, \chi_{1} \wedge \chi_{2}\right\rangle\right\} \\
C S(\lambda, \mathbf{K} \chi) & =C S(\lambda, \chi) \cup\{\langle\lambda, \mathbf{K} \chi\rangle\} \\
C S(\lambda,[\sigma] \chi) & =\left(\bigcup_{p \in \operatorname{dom}(\sigma)} C S(\lambda,(p) \sigma)\right) \cup C S(\lambda \cdot \sigma, \chi) \cup\{\langle\lambda,[\sigma] \chi\rangle\}
\end{aligned}
$$

The set $\operatorname{CS}((), \varphi)$ is the set of contextualised subformulae of $\varphi$.

For example, for $\varphi=[p:=[q:=r] q] p$ we get:

$$
\begin{aligned}
C S((), \varphi) & =C S((),[q:=r] q) \cup\{\langle(p:=[q:=r] q), p\rangle\} \cup\{\langle(),[p:=[q:=r] q] p\rangle\} \\
& =\{\langle(), r\rangle,\langle(q:=r), q\rangle,\langle(),[q:=r] q\rangle\} \cup\{\langle(p:=[q:=r] q), p\rangle,\langle(),[p:=[q:=r] q] p\rangle\}
\end{aligned}
$$

Let $\operatorname{card}(S)$ be the cardinality of a set $S$.

Proposition 9. $-\operatorname{card}(C S(\lambda, \varphi)) \leq \operatorname{len}(\varphi)$.

Proof. - We use induction on the structure of $\varphi$. In the base case $\varphi$ is some atomic formula $p \in P$ :

$$
\operatorname{card}(C S(\lambda, p))=\operatorname{card}(\{\langle\lambda, p\rangle\})=1=\operatorname{len}(p)
$$

In the induction step there are four cases:

(1) $\varphi$ is of the form $\neg \chi$. We have:

$$
\begin{aligned}
\operatorname{card}(C S(\lambda, \neg \chi)) & =\operatorname{card}(C S(\lambda, \chi))+1 \\
& \leq \operatorname{len}(\chi)+1 \\
& =\operatorname{len}(\neg \chi)
\end{aligned}
$$


(2) $\varphi$ is of the form $\chi_{1} \wedge \chi_{2}$. This case is similar to case (1) above and is left to the reader.

(3) $\varphi$ is of the form $\mathbf{K} \chi$. Again, this case is similar to cases (1) and (2) above and is left to the reader.

(4) $\varphi$ is of the form $[\sigma] \chi$. We have:

$$
\begin{aligned}
\operatorname{card}(C S(\lambda,[\sigma] \chi)) & \leq \operatorname{card}\left(\bigcup_{p \in \operatorname{dom}(\sigma)} C S(\lambda,(p) \sigma)\right)+\operatorname{card}(C S(\lambda \cdot \sigma, \chi))+\operatorname{card}(\{\langle\lambda,[\sigma] \chi\rangle\}) \\
& \leq \sum_{p \in \operatorname{dom}(\sigma)} \operatorname{card}(C S(\lambda,(p) \sigma))+\operatorname{card}(C S(\lambda \cdot \sigma, \chi))+1 \\
& \leq \sum_{p \in \operatorname{dom}(\sigma)} \operatorname{len}((p) \sigma)+\operatorname{len}(\chi)+1 \\
& \leq \operatorname{len}(\sigma)+\operatorname{len}(\chi)+1 \\
& =\operatorname{len}([\sigma] \chi)
\end{aligned}
$$

This ends the proof.

We now define the set $B_{\varphi}$ of bi-implications associated to $\varphi$ :

$$
\begin{aligned}
B_{\varphi}= & \left\{n_{p}^{\lambda} \leftrightarrow p \mid\langle\lambda, p\rangle \in C S((), \varphi) \text { and there is no } \lambda_{k} \text { in } \lambda \text { s.th. } p \in \operatorname{dom}\left(\lambda_{k}\right)\right\} \cup \\
& \left\{n_{p}^{\lambda} \leftrightarrow n_{(p) \lambda_{k}}^{\left(\lambda_{1}, \ldots, \lambda_{k-1}\right)} \mid\langle\lambda, p\rangle \in C S((), \varphi) \text { and } \lambda_{k} \text { is the rightmost element of } \lambda \text { s.th. } p \in \operatorname{dom}\left(\lambda_{k}\right)\right\} \cup \\
& \left\{n_{\neg \chi}^{\lambda} \leftrightarrow \neg n_{\chi}^{\lambda} \mid\langle\lambda, \neg \chi\rangle \in C S((), \varphi)\right\} \cup \\
& \left\{n_{\chi_{1} \wedge \chi_{2}}^{\lambda} \leftrightarrow\left(n_{\chi_{1}}^{\lambda} \wedge n_{\chi_{2}}^{\lambda}\right) \mid\left\langle\lambda, \chi_{1} \wedge \chi_{2}\right\rangle \in C S((), \varphi)\right\} \cup \\
& \left\{n_{\mathbf{K} \chi}^{\lambda} \leftrightarrow \mathbf{K} n_{\chi}^{\lambda} \mid\langle\lambda, \mathbf{K} \chi\rangle \in C S((), \varphi)\right\} \cup \\
& \left\{n_{[\sigma] \chi}^{\lambda} \leftrightarrow n_{\chi}^{\lambda \cdot \sigma} \mid\langle\lambda,[\sigma] \chi\rangle \in C S((), \varphi)\right\}
\end{aligned}
$$

It is understood that the propositional letters $n_{\chi}^{\lambda}$ are new for $\varphi$, i.e. they do not to occur in $\varphi$. The next lemma will be useful in the proof of Theorem 13 (cf. Footnote 3).

Lemma 10. - If CS $((), \varphi)$ contains $\langle\lambda, \chi\rangle$ and the bi-implication associated to $n_{\chi}^{\lambda}$ in $B_{\varphi}$ has a right hand side where $n_{\psi}^{\mu}$ occurs then $C S((), \varphi)$ contains $\langle\mu, \psi\rangle$.

Proof. - The only non-trivial case is when $\chi$ is a propositional letter $p$ and $\lambda_{k}$ is the rightmost element of $\lambda$ such that $p \in \operatorname{dom}\left(\lambda_{k}\right)$. In this case, we must show that $\left\langle\left(\lambda_{1}, \ldots, \lambda_{k-1}\right),(p) \lambda_{k}\right\rangle \in C S((), \varphi)$. First, assume that $\lambda=\left(\lambda_{1}, \ldots, \lambda_{k}, \ldots, \lambda_{n}\right)$, where $0 \leq k \leq n$. Now, we have that, by the definition of $C S$, if $\langle\lambda, p\rangle \in$ $C S((), \varphi)$ then it is because $p$ is a sub-formula of some formula $\chi_{n}$ such that $\left\langle\left(\lambda_{1}, \ldots, \lambda_{n-1}\right),\left[\lambda_{n}\right] \chi_{n}\right\rangle \in$ $C S((), \varphi)$. By applying this same argument $n-k$ times, we have that, if $\langle\lambda, p\rangle \in C S((), \varphi)$ then it is because $p$ is a sub-formula of some formula $\chi_{k}$ such that $\left\langle\left(\lambda_{1}, \ldots, \lambda_{k-1}\right),\left[\lambda_{k}\right] \chi_{k}\right\rangle \in C S((), \varphi)$. By hypothesis, $p \in$ $\operatorname{dom}\left(\lambda_{k}\right)$. Then, by the definition of $C S$, we have that the contextualised formula $\left\langle\left(\lambda_{1}, \ldots, \lambda_{k-1}\right),(p) \lambda_{k}\right\rangle \in$ $C S((), \varphi)$.

Finally, the reduction of $\varphi$ is:

$$
\operatorname{red}_{\mathrm{ELA}}(\varphi)=n_{\varphi}^{()} \wedge \mathbf{K}\left(\bigwedge B_{\varphi}\right)
$$


12 Journal of Applied Non-Classical Logics. Volume Volume of the issue undefined - No. Number of the issue undefined/Year of publication undefined

EXAmple 11. - Consider the formula $\varphi=[p:=q][q:=p] q$. It is equivalent to $q$ (which can be checked by the standard reduction method) and is therefore satisfiable. The set of contextualised subformulae of $\varphi$ is computed as follows. First,

$$
C S((p:=q),[q:=p] q)=\{\langle(p:=q), p\rangle,\langle(p:=q, q:=p), q\rangle,\langle(p:=q),[q:=p] q\rangle\}
$$

Second,

$$
\begin{aligned}
C S((), \varphi) & =C S((), q) \cup C S((p:=q),[q:=p] q) \cup\{\langle(),[p:=q][q:=p] q\rangle\} \\
& =\{\langle(), q\rangle\} \cup\{\langle(p:=q), p\rangle,\langle(p:=q, q:=p), q\rangle,\langle(p:=q),[q:=p] q\rangle\} \cup\{\langle(),[p:=q][q:=p] q\rangle\}
\end{aligned}
$$

Now the reduction $\operatorname{red}_{\mathrm{ELA}}(\varphi)$ is

$$
\begin{aligned}
n_{[p:=q][q:=p] q}^{()} \wedge \mathbf{K}( & \left(n_{[p:=q][q:=p] q}^{()} \leftrightarrow n_{[q:=p] q}^{(p:=q)}\right) \wedge \\
& \left(n_{[q:=p] q}^{(p:=q)} \leftrightarrow n_{q}^{(p:=q, q:=p)}\right) \wedge \\
& \left(n_{q}^{(p:=q, q:=p)} \leftrightarrow n_{p}^{(p:=q)}\right) \wedge \\
& \left(n_{p}^{(p:=q)} \leftrightarrow n_{q}^{()}\right) \wedge \\
& \left.\left(n_{q}^{()} \leftrightarrow q\right)\right)
\end{aligned}
$$

Just as the original formula $\varphi$, the reduction of $\varphi$ is satisfiable.

Just as Lutz's reduction, the method above avoids the sub-translation of $\varphi$ and does not lead to an exponential growth of the resultant formula.

Proposition 12. - $\operatorname{red}_{\mathrm{ELA}}$ is a polynomial transformation.

Proof. - By Proposition 9, the set $B_{\varphi}$ contains at most len $(\varphi)$ elements. The length of each new atom $n_{\chi}^{\lambda}$ such that $\langle\lambda, \chi\rangle \in C S((), \varphi)$ is 1 . The maximal length of the right part of a a bi-implication $B_{\varphi}$ is when $\varphi$ is a conjunction. In this case, we have:

$$
\begin{aligned}
\operatorname{len}\left(n_{\chi_{1} \wedge \chi_{2}}^{\lambda} \leftrightarrow\left(n_{\chi_{1}}^{\lambda} \wedge n_{\chi_{2}}^{\lambda}\right)\right) & =\operatorname{len}\left(\left(n_{\chi_{1} \wedge \chi_{2}}^{\lambda} \rightarrow\left(n_{\chi_{1}}^{\lambda} \wedge n_{\chi_{2}}^{\lambda}\right)\right) \wedge\left(\left(n_{\chi_{1}}^{\lambda} \wedge n_{\chi_{2}}^{\lambda}\right) \rightarrow n_{\chi_{1} \wedge \chi_{2}}^{\lambda}\right)\right) \\
& =\operatorname{len}\left(\neg\left(n_{\chi_{1} \wedge \chi_{2}}^{\lambda} \wedge \neg\left(n_{\chi_{1}}^{\lambda} \wedge n_{\chi_{2}}^{\lambda}\right)\right) \wedge \neg\left(\left(n_{\chi_{1}}^{\lambda} \wedge n_{\chi_{2}}^{\lambda}\right) \wedge \neg n_{\chi_{1} \wedge \chi_{2}}^{\lambda}\right)\right) \\
& =15
\end{aligned}
$$

This therefore bounds the length of $\wedge B_{\varphi}$ : len $(\varphi)$ times the worst case length 15 of each element in that set, plus the conjunction symbols, i.e., $16 \times \operatorname{len}(\varphi)-1$. Therefore, $\operatorname{red}_{\mathrm{ELA}}$ is a polynomial transformation from the language of ELA to that of EL; precisely, the length of $\operatorname{red}_{\mathrm{ELA}}(\varphi)=n_{\varphi}^{()} \wedge \mathbf{K}\left(\wedge B_{\varphi}\right)$ is bound by $3+(16 \times \operatorname{len}(\varphi)-1)$

Intuitively, for each pair $\langle\lambda, \chi\rangle \in C S((), \varphi)$, the associated bi-implication guarantees that the new propositional letter $n_{\chi}^{\lambda}$ is true in a pointed model $(M, w)$ exactly when $\chi$ is true in the pointed model $\left(M^{\lambda}, w\right)$. We then have the following result:

THeOrem 13. - For every ELA formula $\varphi_{0}$ :

1) $\operatorname{red}_{\mathrm{ELA}}\left(\varphi_{0}\right)$ is an EL formula.

2) $\varphi_{0}$ is ELA satisfiable if and only if $\operatorname{red}_{\mathrm{ELA}}\left(\varphi_{0}\right)$ is EL satisfiable. 
Proof. - First, $\operatorname{red}_{\mathrm{ELA}}\left(\varphi_{0}\right)$ is clearly an EL formula.

Let us prove the "if" part of the second statement: suppose $M, w_{0} \vDash n_{\varphi_{0}}^{(} \wedge \mathbf{K}\left(\wedge B_{\varphi_{0}}\right)$. We show by induction on $\operatorname{len}(\lambda)+\operatorname{len}(\varphi)$ that

$$
\text { for every }(\lambda, \chi) \in C S\left((), \varphi_{0}\right) \text { and } w \in R^{\lambda}\left(w_{0}\right): M, w \vDash n_{\chi}^{\lambda} \text { iff } M^{\lambda}, w \vDash \chi
$$

where len $(\lambda)$ and $M^{\lambda}$ are recursively defined as expected as: $\operatorname{len}(())=0$, and $\operatorname{len}(\lambda \cdot \sigma)=\operatorname{len}(\lambda)+\operatorname{len}(\sigma)$; and $M^{()}=M$ and $M^{\lambda \cdot \sigma}=\left(M^{\lambda}\right)^{\sigma}$. Note that since $\left((), \varphi_{0}\right) \in C S\left((), \varphi_{0}\right)$ this allows us to conclude that $M, w_{0} \vDash \varphi_{0}$.

The induction base is $\operatorname{len}(\lambda)+\operatorname{len}(\varphi)=1$. Therefore, we must have $\lambda=()$ and $\varphi=p$ for some propositional letter $p$ occurring in $\varphi_{0}$. Then for every $w \in R^{\lambda}\left(w_{0}\right)$ we have $M, w \vDash p$ iff $M, w \vDash n_{p}^{(}$, because by hypothesis $M, w \vDash B_{\varphi}$ and $n_{p}^{()} \leftrightarrow p \in B_{\varphi}$.

For the induction step, suppose $M^{\lambda}, w \vDash \chi$ iff $M, w \vDash n_{\chi}^{\lambda}$ for every $w$ in $R^{\lambda}\left(w_{0}\right)$ and $\langle\mu, \chi\rangle$ such that $\operatorname{len}(\mu)+\operatorname{len}(\chi)<m$, and let $\langle\lambda, \varphi\rangle \in C S\left((), \varphi_{0}\right)$ with $\operatorname{len}(\lambda)+\operatorname{len}(\varphi)=m$. Let $\lambda$ be $\left(\lambda_{1}, \ldots, \lambda_{n}\right)$. We analyse the form of $\varphi$.

(1) $\varphi=p \in P$.

We consider two sub-cases.

- If there is no $k \leq n$ such that $p \in \operatorname{dom}\left(\lambda_{k}\right)$, then

$M, w \vDash n_{p}^{\lambda}$

iff $M, w \models p$

iff $w \in V(p)$

(because $M, w \vDash B_{\varphi}$ )

iff $w \in V^{\lambda}(p)$

iff $M^{\lambda}, w \models p$.

(because there is no $k$ s.th. $p \in \operatorname{dom}\left(\lambda_{k}\right)$ )

- If there exists $k \leq n$ such that $p \in \operatorname{dom}\left(\lambda_{k}\right)$ then consider the rightmost such $k$, i.e. such that $p \notin \operatorname{dom}\left(\lambda_{l}\right)$ for every $l$ such that $k<l \leq n$. Then

$M, w \vDash n_{p}^{\lambda}$

iff $M, w \vDash n_{(p) \lambda_{k}}^{\left(\lambda_{1}, \ldots, \lambda_{k-1}\right)}$

iff $M^{\left(\lambda_{1}, \ldots, \lambda_{k-1}\right)}, w \models(p) \lambda_{k}$

(because $M, w \vDash B_{\varphi}$ )

iff $M^{\left(\lambda_{1}, \ldots, \lambda_{k-1}\right)}, w \models\left[\lambda_{k}\right] p$

(by I.H. ${ }^{3}$ )

iff $M^{\left(\lambda_{1}, \ldots, \lambda_{k}\right)}, w \vDash p$

iff $M^{\lambda}, w \models p$

(2) $\varphi=\neg \psi$.

$M, w \vDash n_{\neg \psi}^{\lambda}$

iff $M, w \models \neg n_{\psi}^{\lambda}$

iff $M, w \not \models n_{\psi}^{\lambda}$

iff $M^{\lambda}, w \not \neq \psi$

iff $M^{\lambda}, w \models \neg \psi$.

(3) $\varphi=\psi_{1} \wedge \psi_{2}$.

This is similar to case (2) above and is left to the reader.

(4) $\varphi=\mathbf{K} \psi$.

$M, w \models n_{\mathbf{K} \psi}^{\lambda}$

iff $M, w \vDash \mathbf{K} n_{\psi}^{\lambda}$

(because $M, w \vDash B_{\varphi}$ )

3. The induction hypothesis is applicable because $\operatorname{len}\left(\left(\lambda_{1}, \ldots, \lambda_{k-1}\right)+\operatorname{len}\left((p) \lambda_{k}\right)<\operatorname{len}\left(\left(\lambda_{1}, \ldots, \lambda_{k-1}\right)+\operatorname{len}\left(\lambda_{k}\right)<\right.\right.$ $\operatorname{len}(\lambda)+\operatorname{len}(p)$, and because $\left\langle\left(\lambda_{1}, \ldots, \lambda_{k-1}\right),(p) \lambda_{k}\right\rangle \in C S\left((), \varphi_{0}\right)$. The latter is guaranteed by Lemma 10 . 
14 Journal of Applied Non-Classical Logics. Volume Volume of the issue undefined - No. Number of the issue undefined/Year of publication undefined

iff $M, u \models n_{\psi}^{\lambda}$ for all $u \in R(w)$

iff $M^{\lambda}, u \models \psi$ for all $u \in R^{\lambda}(w)$

(by I.H. and because $R^{\lambda}=R$ )

iff $M^{\lambda}, w \models \mathbf{K} \psi$.

(5) $\varphi=[\sigma] \psi$.

$M, w \vDash n_{[\sigma] \psi}^{\lambda}$

iff $M, w \models n_{\psi}^{\lambda \cdot \sigma}$

(because $M, w \models B_{\varphi}$ )

iff $M^{\lambda \cdot \sigma}, w \models \psi$

(by I.H. ${ }^{4}$ )

iff $M^{\lambda}, w \models[\sigma] \psi$.

This ends the "if" part.

Finally, let us prove the "only if" part: suppose $M=\langle W, R, V\rangle$ and $M, w_{0} \vDash \varphi_{0}$. We construct a new model $M^{\prime}=\left\langle W, R, V^{\prime}\right\rangle$, where $V^{\prime}$ is defined as follows: $V^{\prime}(p)=V(p)$ if $p$ occurs in $\varphi_{0}$, and $V^{\prime}\left(n_{\chi}^{\lambda}\right)=\left\{w \mid M^{\lambda}, w \vDash \chi\right\}$, for $\langle\lambda, \chi\rangle \in C S\left((), \varphi_{0}\right)$.

We clearly have $M^{\prime}, w_{0} \vDash n_{\varphi_{0}}^{()}$. It remains to show that:

$$
M^{\prime}, w \vDash \chi \text {, for every } \chi \in B_{\varphi_{0}} \text { and every } w \in W
$$

Let $\lambda$ be $\left(\lambda_{1}, \ldots, \lambda_{n}\right)$. We inspect the possible forms of $\chi$.

(1) $\chi=p \in P$.

We consider two sub-cases.

- If there is no $k \leq n$ such that $p \in \operatorname{dom}\left(\lambda_{k}\right)$, then:

$M^{\prime}, w \vDash n_{p}^{\lambda}$

iff $w \in V^{\prime}\left(n_{p}^{\lambda}\right)$

iff $M^{\lambda}, w=p$

(by the definition of $V^{\prime}$ )

iff $w \in V^{\lambda}(p)$

iff $w \in V(p)$

(because $p \notin \operatorname{dom}\left(\lambda_{k}\right)$ for any $k$ )

iff $w \in V^{\prime}(p)$

(by the definition of $V^{\prime}$ )

iff $M^{\prime}, w \vDash p$.

Therefore, $M^{\prime}, w \vDash n_{p}^{\lambda} \leftrightarrow p$.

- If there exists $k \leq n$ such that $p \in \operatorname{dom}\left(\lambda_{k}\right)$ then consider the rightmost such $k$, i.e. such that $p \notin \operatorname{dom}\left(\lambda_{l}\right)$ for every $l$ such that $k<l \leq n$. Then:

$M^{\prime}, w \vDash n_{p}^{\lambda}$

iff $w \in V^{\prime}\left(n_{p}^{\lambda}\right)$

iff $M^{\lambda}, w=p$

(by the definition of $V^{\prime}$ )

iff $w \in V^{\lambda}(p)$

iff $w \in V^{\left(\lambda_{1}, \cdots, \lambda_{k}\right)}(p)$

(because $p \notin \operatorname{dom}\left(\lambda_{l}\right)$, for $k<l \leq n$ )

iff $M^{\left(\lambda_{1}, \cdots, \lambda_{k}\right)}, w \models p$

iff $M^{\left(\lambda_{1}, \cdots, \lambda_{k-1}\right)}, w \models\left[\lambda_{k}\right] p$

iff $M^{\left(\lambda_{1}, \cdots, \lambda_{k-1}\right)}, w \models(p) \lambda_{k}$

(by the reduction axiom)

iff $M^{\prime}, w \vDash n_{(p) \lambda_{k}}^{\left(\lambda_{1}, \ldots, \lambda_{k-1}\right)}$

(by the definition of $V^{\prime}$ ).

Therefore, $M^{\prime}, w \vDash n_{p}^{\lambda} \leftrightarrow n_{(p) \lambda_{k}}^{\left(\lambda_{1}, \ldots, \lambda_{k-1}\right)}$.

(2) $\chi=\neg \chi_{1}$.

$M^{\prime}, w \vDash n_{\neg \chi_{1}}^{\lambda}$ iff $M^{\lambda}, w \vDash \neg \chi_{1}$

(by the definition of $V^{\prime}$ )

4. The induction hypothesis is applicable because $\operatorname{len}(\lambda \cdot \sigma)+\operatorname{len}(\psi)<\operatorname{len}(\lambda)+\operatorname{len}([\sigma] \psi)$. 
iff $M^{\lambda}, w \not \neq \chi_{1}$

iff $M^{\prime}, w \not=n_{\chi_{1}}^{\lambda}$

iff $M^{\prime}, w \vDash \neg n_{\chi_{1}}^{\lambda}$.

(again, by the definition of $V^{\prime}$ )

Therefore, $M^{\prime}, w \vDash n_{\neg \chi_{1}}^{\lambda} \leftrightarrow \neg n_{\chi_{1}}^{\lambda}$.

(3) $\chi=\chi_{1} \wedge \chi_{2}$.

This is similar to case (2) above and left to the reader.

(4) $\chi=\mathbf{K}_{\chi_{1}}$.

$M^{\prime}, w \vDash n_{\mathbf{K}_{\chi}}^{\lambda}$

iff $M^{\lambda}, w \models \mathbf{K} \chi_{1}$

iff $M^{\lambda}, u \vDash \chi_{1}$ for all $u \in R^{\lambda}(w)$

iff $M^{\prime}, u=n_{\chi_{1}}^{\lambda}$ for all $u \in R^{\lambda}(w)$

iff $M^{\prime}, u \vDash n_{\chi_{1}}^{\lambda}$ for all $u \in R(w)$

(by the definition of $V^{\prime}$ )

iff $M^{\prime}, w \models \mathbf{K} n_{\chi}^{\lambda}$.

(5) $\chi=[\sigma] \chi_{1}$

$M^{\prime}, w \vDash n_{[\sigma] \chi_{1}}^{\lambda}$

iff $M^{\lambda}, w=[\sigma] \chi_{1}$

(by the definition of $V^{\prime}$ )

iff $M^{\lambda \cdot \sigma}, w \vDash \chi_{1}$

iff $M^{\prime}, w \vDash n_{\chi 1}^{\lambda \cdot \sigma}$

(again by the definition of $V^{\prime}$ )

(because $R^{\lambda}=R$ )

This ends the "only if" part.

THEOREM 14. - The problem of deciding satisfiability for single-agent epistemic logic with assignments ELA is NP-complete.

PRoof. - By Proposition 12 red $_{\text {ELA }}$ is a polynomial transformation from the language of ELA to that of EL. Moreover, red ELA $_{\text {p }}$ preserves satisfiability because of Theorem 13. Therefore, the complexity of the problem of deciding ELA satisfiability is at most that of deciding single-agent EL satisfiability, which is in NP.

Due to Proposition 2, ELA is a conservative extension of EL. As the problem of deciding EL satisfiability is NP-hard, the problem of deciding ELA satisfiability is also NP-hard. It follows that the problem of deciding ELA satisfiability is NP-complete.

\subsection{From PALA to EL}

Now, we extend the procedure given in the last section to the entire logic PALA. The first thing to do is to extend contexts to sequences of assignments and announcements.

Definition 15. - The set CS of contextualised subformulae is the same as in Definition 8 plus the following clause for announcements:

$$
\operatorname{CS}\left(\lambda,\left[! \chi_{1}\right] \chi_{2}\right)=\operatorname{CS}\left(\lambda, \chi_{1}\right) \cup \operatorname{CS}\left(\lambda \cdot ! \chi_{1}, \chi_{2}\right) \cup\left\{\left\langle\lambda,\left[! \chi_{1}\right] \chi_{2}\right\rangle\right\}
$$

Proposition 16. - Let $\varphi$ be a PALA formula. Then $\operatorname{card}(C S(\lambda, \varphi)) \leq \operatorname{len}(\varphi)$.

Proof. - We employ induction on the structure of $\varphi$. The induction base, as well as cases (1) to (4) are exactly as in the proof of Proposition 9. In the induction step we have an additional case: 
16 Journal of Applied Non-Classical Logics. Volume Volume of the issue undefined - No. Number of the issue undefined/Year of publication undefined

(5) $\varphi=\left[\chi_{1}\right] \chi_{2}$.

$\operatorname{card}\left(C S\left(\lambda,\left[! \chi_{1}\right] \chi_{2}\right)\right)$

$\leq \operatorname{card}\left(C S\left(\lambda, \chi_{1}\right)\right)+\operatorname{card}\left(C S\left(\lambda \cdot ! \chi_{1}, \chi_{2}\right)\right)+\operatorname{card}\left(\left\{\left\langle\lambda,\left[! \chi_{1}\right] \chi_{2}\right\rangle\right\}\right)$

$\leq \operatorname{len}\left(\chi_{1}\right)+\operatorname{len}\left(\chi_{2}\right)+1$

(by I.H.)

$=\operatorname{len}\left(\left[! \chi_{1}\right] \chi_{2}\right)$.

This ends the proof.

We define the set $B_{\varphi}$ of bi-implications as follows:

$$
\begin{aligned}
B_{\varphi}= & \left\{n_{p}^{\lambda} \leftrightarrow p \mid\langle\lambda, p\rangle \in C S((), \varphi) \text { and there is no } \lambda_{k} \text { in } \lambda \text { s.th. } p \in \operatorname{dom}\left(\lambda_{k}\right)\right\} \cup \\
& \left\{n_{p}^{\lambda} \leftrightarrow n_{(p) \lambda_{k}}^{\left(\lambda_{1}, \ldots, \lambda_{k-1}\right)} \mid\langle\lambda, p\rangle \in C S((), \varphi) \text { and } \lambda_{k} \text { is the rightmost element of } \lambda \text { s.th. } p \in \operatorname{dom}\left(\lambda_{k}\right)\right\} \cup \\
& \left\{n_{\neg \chi}^{\lambda} \leftrightarrow \neg n_{\chi}^{\lambda} \mid\langle\lambda, \chi\rangle \in C S((), \varphi)\right\} \cup \\
& \left\{n_{\chi_{1} \wedge \chi_{2}}^{\lambda} \leftrightarrow\left(n_{\chi_{1}}^{\lambda} \wedge n_{\chi_{2}}^{\lambda}\right) \mid\left\langle\lambda, \chi_{1} \wedge \chi_{2}\right\rangle \in C S((), \varphi)\right\} \cup \\
& \left.\left\{n_{\mathbf{K} \chi}^{\lambda} \leftrightarrow \mathbf{K}\left(\bigwedge_{k \leq n, \lambda_{k}=! \psi} n_{\psi}^{\left(\lambda_{1}, \ldots, \lambda_{k-1}\right)}\right) \rightarrow n_{\chi}^{\lambda}\right) \mid\langle\lambda, \mathbf{K} \chi\rangle \in C S((), \varphi) \text { and } \lambda \text { is of length } n\right\} \cup \\
& \left\{n_{[\sigma] \chi}^{\lambda} \leftrightarrow n_{\chi}^{\lambda \cdot \sigma} \mid\langle\lambda,[\sigma] \chi\rangle \in C S((), \varphi)\right\} \cup \\
& \left\{n_{\left[! \chi_{1} \mid \chi_{2}\right.}^{\lambda} \leftrightarrow\left(n_{\chi_{1}}^{\lambda} \rightarrow n_{\chi_{2}}^{\lambda \cdot\left(\chi_{1}\right)}\right) \mid\left\langle\lambda,\left[! \chi_{1}\right] \chi_{2}\right\rangle \in C S((), \varphi)\right\}
\end{aligned}
$$

where we extend the domain function dom to announcements by stipulating $\operatorname{dom}(! \varphi)=\emptyset$. The clause for $\mathbf{K}$ conditions the abbreviation of $\chi$ by the contextual truth of all the preconditions $\psi$ occurring in the context $\lambda$ (precisely, of the abbreviations of these preconditions).

Finally, the reduction of the PALA formula $\varphi$ is the formula

$$
\operatorname{red}_{\mathrm{PALA}}(\varphi)=n_{\varphi}^{0} \wedge \mathbf{K}\left(\bigwedge B_{\varphi}\right)
$$

Example 17. - Consider the formula $\varphi=[! \neg p][q:=p] \mathbf{K} q$. Applying the reduction axioms we get:

$$
\begin{aligned}
{[! \neg p][q:=p] \mathbf{K} q } & \leftrightarrow[! \neg p] \mathbf{K}[q:=p] q \\
& \leftrightarrow[! \neg p] \mathbf{K} p \\
& \leftrightarrow \neg p \rightarrow \mathbf{K}[! \neg p] p \\
& \leftrightarrow \neg p \rightarrow \mathbf{K}(\neg p \rightarrow p) \\
& \leftrightarrow \neg p \rightarrow \mathbf{K} p
\end{aligned}
$$

The last formula is EL equivalent to $p$. Therefore, $\varphi$ is PALA satisfiable. 
First, we compute the set of contextualised subformulae. We have:

$$
\begin{aligned}
C S((),[! \neg p][q:=p] \mathbf{K} q)= & C S((), \neg p) \cup C S((! \neg p),[q:=p] \mathbf{K} q) \cup\{\langle(),[! \neg p][q:=p] \mathbf{K} q\rangle\} \\
= & \{\langle(), \neg p\rangle,\langle(), p\rangle\} \cup C S((! \neg p), p) \cup C S((! \neg p, q:=p), \mathbf{K} q) \cup \\
& \{\langle(! \neg p),[q:=p] \mathbf{K} q\rangle\} \cup\{\langle(),[! \neg p][q:=p] \mathbf{K} q\rangle\} \\
= & \{\langle(), \neg p\rangle,\langle(), p\rangle\} \cup\{\langle(! \neg p), p\rangle\} \cup\{\langle(! \neg p, q:=p), q\rangle,\langle(! \neg p, q:=p), \mathbf{K} q\rangle\} \cup \\
& \{\langle(! \neg p),[q:=p] \mathbf{K} q\rangle\} \cup\{\langle(),[! \neg p][q:=p] \mathbf{K} q\rangle\}
\end{aligned}
$$

Now, using the bi-implications, the reduction of $\varphi$ is

$$
\begin{aligned}
n_{[! \neg p][q:=p] \mathbf{K} q}^{()} \wedge \mathbf{K} & \left(\left(n_{[! \neg p][q:=p] \mathbf{K} q}^{()} \leftrightarrow\left(n_{\neg p}^{()} \rightarrow n_{[q:=p] \mathbf{K} q}^{(! \neg p)}\right)\right) \wedge\right. \\
& \left(n_{[q:=p] \mathbf{K} q}^{(! \neg p)} \leftrightarrow n_{\mathbf{K} q}^{(! \neg p, q:=p)}\right) \wedge \\
& \left(n_{\mathbf{K} q}^{(! \neg p, q:=p)} \leftrightarrow \mathbf{K}\left(n_{\neg p}^{()} \rightarrow n_{q}^{(! \neg p, q:=p)}\right)\right) \wedge \\
& \left(n_{q}^{(! \neg p, q:=p)} \leftrightarrow n_{p}^{(! \neg p)}\right) \wedge \\
& \left(n_{p}^{(! \neg p)} \leftrightarrow p\right) \wedge \\
& \left(n_{\neg p}^{()} \leftrightarrow \neg n_{p}^{()}\right) \wedge \\
& \left.\left(n_{p}^{()} \leftrightarrow p\right)\right)
\end{aligned}
$$

It can be checked that the reduction of $\varphi$ is EL satisfiable.

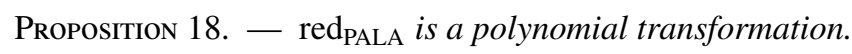

Proof. - First, due to Proposition 16, $\operatorname{card}(C S(\lambda, \varphi)) \leq \operatorname{len}(\varphi)$. Second, the longest bi-implications are those of the form

$$
n_{\mathbf{K} \chi}^{\lambda} \leftrightarrow \mathbf{K}\left(\left(\bigwedge_{k \leq n, \lambda_{k}=! \psi} n_{\psi_{k}}^{\left(\lambda_{1}, \ldots, \lambda_{k-1}\right)}\right) \rightarrow n_{\chi}^{\lambda}\right)
$$

As $n$ is at most len $(\varphi)$, the length of the conjunction $\bigwedge_{k \leq n, \lambda_{k}=! \psi} n_{\psi_{k}}^{\left(\lambda_{1}, \ldots, \lambda_{k-1}\right)}$ is at most $2 \times \operatorname{len}(\varphi)-1$. Hence, the length of that bi-implication itself is at most $7+(2 \times 1)+2 \times(2 \times \operatorname{len}(\varphi)-1+5))=17+(4 \times \operatorname{len}(\varphi))$. Therefore, the length of each bi-implication is linear in len $(\varphi)$.

It follows that the length of $\operatorname{red}_{\mathrm{PALA}}(\varphi)$ is quadratic in $\operatorname{len}(\varphi)$.

THEOREM 19. - For every PALA formula $\varphi_{0}$ :

1) $\operatorname{red}_{\mathrm{PALA}}\left(\varphi_{0}\right)$ is an EL formula.

2) $\varphi_{0}$ is PALA satisfiable if and only if $\operatorname{red}_{\mathrm{PALA}}\left(\varphi_{0}\right)=n_{\varphi_{0}}^{()} \wedge \mathbf{K}\left(\wedge B_{\varphi_{0}}\right)$ is EL satisfiable.

Proof. - First, $\operatorname{red}_{\mathrm{PALA}}\left(\varphi_{0}\right)$ is clearly an EL formula.

For the "if" part of the second statement suppose $M, w_{0} \vDash n_{\varphi_{0}}^{()} \wedge \mathbf{K}\left(\wedge B_{\varphi_{0}}\right)$. We show by induction on $\operatorname{len}\left(\lambda_{1}\right)+\ldots+\operatorname{len}\left(\lambda_{n}\right)+\operatorname{len}(\chi)$ that for every $\left(\left(\lambda_{1}, \ldots, \lambda_{n}\right), \chi\right) \in C S\left((), \varphi_{0}\right)$ :

if $M^{\left(\lambda_{1}, \ldots, \lambda_{k-1}\right)}, w_{0}=\psi_{k}$ for all $\lambda_{k}$ s.th. $\lambda_{k}=! \psi_{k}$, and $w \in R^{\left(\lambda_{1}, \ldots, \lambda_{k-1}\right)}\left(w_{0}\right)$, then $M, w \vDash n_{\chi}^{\lambda}$ iff $M^{\lambda}, w \vDash \chi$

where the definition of length is extended to announcements by stipulating len $(! \varphi)=\operatorname{len}(\varphi)$. Since $\left((), \varphi_{0}\right) \in C S\left((), \varphi_{0}\right)$ this allows us to conclude that $M, w_{0} \vDash \varphi_{0}$, i.e. that $\varphi_{0}$ is PALA satisfiable. 
In the induction base $\lambda=()$ and $\chi=p$, for some $p$ in $\varphi_{0}$. Then we have $M, w \vDash n_{p}^{()}$iff $M, w \vDash p$ because $M, w \vDash B_{\varphi}$ and because $w \in R^{()}\left(w_{0}\right)$.

In the induction step, let $\lambda$ be $\left(\lambda_{1}, \ldots, \lambda_{n}\right)$, for some $n \geq 0$, and suppose $M^{\left(\lambda_{1}, \ldots, \lambda_{k-1}\right)}, w_{0} \vDash \psi_{k}$ if $\lambda_{k}=! \psi_{k}$. We analyse the form of $\varphi$. There are six cases. Cases (1) to (3) and (5) are exactly as in the "if" part of proof of Theorem 13. Case (4) has to be adapted:

(4) $\chi=\mathbf{K} \chi_{1}$.

$M, w \vDash n_{\mathbf{K} \chi_{1}}^{\lambda}$

iff $M, w \vDash \mathbf{K}\left(\left(\bigwedge_{k \leq n, \lambda_{k}=! \varphi_{k}} n_{\psi_{k}}^{\left(\lambda_{1}, \ldots, \lambda_{k-1}\right)}\right) \rightarrow n_{\chi_{1}}^{\lambda}\right)$

(because $M, w_{0} \vDash B_{\varphi_{0}}$ and $w \in R^{\lambda}\left(w_{0}\right)$ ) iff for all $u \in R(w), M, u=n_{\psi_{k}}^{\left(\lambda_{1}, \ldots \lambda_{k-1}\right)}$ for all $k \leq n$ s.th. $\lambda_{k}=! \psi_{k}$, implies $M, u \vDash n_{\chi_{1}}^{\lambda}$ iff for all $u \in R(w), M^{\left(\lambda_{1}, \ldots \lambda_{k-1}\right)}, u \models \psi_{k}$ for all $k \leq n$ s.th. $\lambda_{k}=! \psi_{k}$, implies $M^{\lambda}, u \models \chi_{1}$

iff for all $u \in R^{\lambda}(w), M^{\lambda}, u \models \chi_{1}$

(by I.H., $n$ times) ${ }^{5}$ (because $u \in R^{\lambda}(w)$ iff iff $M^{\lambda}, w \models \mathbf{K}_{1}$

Case (6) is new:

(6) $\chi=\left[! \chi_{1}\right] \chi_{2}$

$M, w \vDash n_{\left[! \chi_{1}\right] \chi_{2}}^{\lambda}$

iff $M, w \models n_{\chi_{1}}^{\lambda} \rightarrow n_{\chi_{2}}^{\lambda ! ! \chi_{1}}$

iff $M, w \not=n_{\chi_{1}}^{\lambda}$, or $M, w=n_{\chi_{1}}^{\lambda}$ and $M, w \vDash n_{\chi_{2}}^{\lambda \cdot ! \psi_{1}}$

(because $M, w \vDash B_{\varphi_{0}}$ )

iff $M^{\lambda}, w \vDash \chi_{1}$, or $M^{\lambda}, w \vDash \chi_{1}$ and $M, w \vDash n_{\chi_{2}}^{\lambda ! \psi_{1}}$

(by I.H. on $\chi_{1}$ )

iff $M^{\lambda}, w \vDash \chi_{1}$, or $M^{\lambda}, w \vDash \chi_{1}$ and $M^{\lambda \cdot ! \psi_{1}}, w \vDash \chi_{2}$

(by I.H. on $\left.\chi_{2}\right)^{7}$

iff $M^{\lambda}, w \vDash\left[! \chi_{1}\right] \chi_{2}$

This ends the "if" part.

Let us now prove the "only if" part. Let $M=\langle W, R, V\rangle$ and $M, w_{0} \vDash \varphi_{0}$. We define a new model $M^{\prime}=\left\langle W, R, V^{\prime}\right\rangle$, where $V^{\prime}$ is defined as follows: $V^{\prime}(p)=V(p)$ if $p$ is in $\varphi_{0}$, and $V^{\prime}\left(n_{\chi}^{\lambda}\right)=\left\{w \mid M^{\lambda}, w \vDash \chi\right\}$ for $(\lambda, \chi) \in C S\left((), \varphi_{0}\right)$. We show that:

$$
M^{\prime}, w \vDash B_{\varphi_{0}} \text {, for all } w \in W
$$

There are six cases, according to the form of the left hand sides of $B_{\varphi_{0}}$. Cases (1) to (3) and (5) are exactly as in the "only if" part of the proof of Theorem 13, so we only prove the remaining two cases.

5. The induction hypothesis applies to every $\psi_{k}$ because

$$
\begin{aligned}
\operatorname{len}\left(\lambda_{1}\right)+\ldots+\operatorname{len}\left(\lambda_{k-1}\right)+\operatorname{len}\left(\psi_{k}\right) & =\operatorname{len}\left(\lambda_{1}\right)+\ldots+\operatorname{len}\left(\lambda_{k-1}\right)+\operatorname{len}\left(\lambda_{k}\right) \\
& \leq \operatorname{len}\left(\lambda_{1}\right)+\ldots+\operatorname{len}\left(\lambda_{n}\right) \\
& <\operatorname{len}\left(\lambda_{1}\right)+\ldots+\operatorname{len}\left(\lambda_{n}\right)+\operatorname{len}\left(\chi_{1}\right)
\end{aligned}
$$

6. In detail, we prove by induction on $k$ that $u \in R^{\left(\lambda_{1}, \ldots \lambda_{k}\right)}(w)$ iff $u \in R^{\left(\lambda_{1}, \ldots \lambda_{k-1}\right)}(w)$ and $\lambda_{k}=! \psi_{k}$ implies $M^{\left(\lambda_{1}, \ldots \lambda_{k-1}\right)}, u \vDash$ $\psi_{k}$.

7. Note that the induction hypothesis applies to $\chi_{2}$ because due to $M^{\lambda}, w \vDash \chi_{1}$, the condition of the induction hypothesis is satisfied. 
(4) $\chi=\mathbf{K} \chi_{1}$.

Let $\lambda$ be $\left(\lambda_{1}, \ldots, \lambda_{n}\right)$.

$M^{\prime}, w \vDash n_{\mathbf{K}_{\chi_{1}}}^{\lambda}$

iff $M^{\lambda}, w \models \mathbf{K} \chi_{1}$

(by the definition of $V^{\prime}$ )

iff for all $u \in R^{\lambda}(w), M^{\lambda}, u \vDash \chi_{1}$

iff for all $u \in R(w), M^{\left(\lambda_{1}, \cdots, \lambda_{k-1}\right)}, u \models \psi_{k}$ for all $k \leq n$ s.th. $\lambda_{k}=\psi_{k}$ implies $M^{\lambda}, u \models \chi_{1}$

(because $u \in R^{\lambda}(w)$ iff $u \in R(w)$ and $M^{\left(\lambda_{1}, \ldots \lambda_{k-1}\right)}, u \models \psi_{k}$ for every $\left.k \leq n\right)^{8}$

iff for all $u \in R(w), M^{\prime}, u \models n_{\psi_{k}}^{\left(\lambda_{1}, \cdots, \lambda_{k-1}\right)}$ for all $k \leq n$ s.th. $\lambda_{k}=! \psi_{k}$ implies $M^{\prime}, u \models n_{\chi_{1}}^{\lambda}$

iff $M^{\prime}, w \vDash \mathbf{K}\left(\left(\bigwedge_{k \leq n} n_{\psi_{k}}^{\lambda_{1}, \ldots, \lambda_{k-1}}\right) \rightarrow n_{\chi_{1}}^{\lambda}\right)$.

(again by the definition of $V^{\prime}$ )

(6) $\chi=\left[! \chi_{1}\right] \chi_{2}$

$M^{\prime}, w \vDash n_{\left[! \chi_{1}\right] \chi_{2}}^{\lambda}$

iff $M^{\lambda}, w \vDash\left[! \chi_{1}\right] \chi_{2}$

(by the definition of $V^{\prime}$ )

iff $M^{\lambda}, w \vDash \chi_{1}$ implies $M^{\lambda \cdot \chi_{1}}, w \vDash \chi_{2}$

iff $M^{\prime}, w \models n_{\chi_{1}}^{\lambda}$ implies $M^{\prime}, w \models n_{\chi_{2}}^{\lambda \cdot \chi_{1}}$

iff $M^{\prime}, w \vDash n_{\chi_{1}}^{\lambda} \rightarrow n_{\chi_{2}}^{\lambda \cdot \chi_{1}}$

(again by the definition of $V^{\prime}$ )

This ends the "only if" part (and the proof).

THEOREM 20. - The problem of deciding satisfiability for single-agent public announcement logic with assignment is NP-complete.

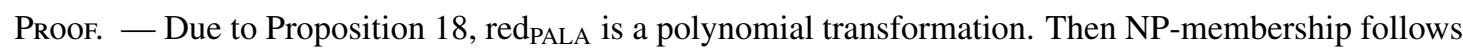
from Theorem 19. Finally, NP-hardness follows from the fact that PALA is a conservative extension of EL (Proposition 2).

\section{Multiagent case}

As said before, multiagent PALA is a straightforward extension of PALA. Assume a finite non-empty set $N$ of agents. Then, in the language of multiagent PALA, instead of a single epistemic operator $\mathbf{K}$, we define an operator $\mathbf{K}_{i}$ for each agent $i \in N$. Formulae of the form $\mathbf{K}_{i} \varphi$ are read "agent $i$ knows that $\varphi$ ". The length of such formulas is defined as usual: $\operatorname{len}\left(\mathbf{K}_{i} \varphi\right)=1+\operatorname{len}(\varphi)^{9}$.

For its semantics, the models are tuples $M=\langle W, R, V\rangle$, where $W$ and $V$ are as for single-agent PALA and where $R: N \longrightarrow 2^{W \times W}$ associates to each agent $i$ an equivalence relation $R_{i}$. The satisfaction relation is as before for the Boolean and the dynamic operators, and:

$$
M, w \vDash \mathbf{K}_{i} \varphi \quad \quad \quad \text { iff } \quad R_{i}(w) \subseteq \llbracket \varphi \rrbracket_{M}
$$

The technique we used to provide an optimal transformation from single-agent PALA to single-agent EL cannot be taken over literally in the multiagent case. The reason is simple. Note that the key idea of the reduction is that each bi-implication in $B_{\varphi}$ is enforced to be true in every world of the model (assuming

8. In detail, we prove by induction on $k$ that $u \in R^{\left(\lambda_{1}, \ldots \lambda_{k}\right)}(w)$ iff $u \in R^{\left(\lambda_{1}, \ldots \lambda_{k-1}\right)}(w)$ and $\lambda_{k}=! \psi_{k}$ implies $M^{\left(\lambda_{1}, \ldots \lambda_{k-1}\right)}, u \models$ $\psi_{k}$.

9. Strictly speaking, we also have to encode the length of the agent index of $\mathbf{K}$. We suppose nevertheless that $\operatorname{len}\left(\mathbf{K}_{i} \varphi\right)=1+\operatorname{len}(\varphi)$ for the same reasons as we gave in Footnote 2 of Section 2.1. 
20 Journal of Applied Non-Classical Logics. Volume Volume of the issue undefined - No. Number of the issue undefined/Year of publication undefined

that it is point-generated). In the single-agent case, the operator $\mathbf{K}$ is enough to enforce that, but it is clearly not enough in the multiagent case.

There is a simple way to get around this problem. All that we need is a kind of master modality. That role could be played by the common knowledge operator: if one replaces the operator $\mathbf{K}$ by that operator in theorems 13 and 19 then we obtain the same results. In this case, the reduction would be from multiagent PALA without common knowledge to multiagent EL with common knowledge. But the latter is EXPTIME complete, while multiagent PALA without common knowledge is PSPACE complete, as we show in the sequel.

To do so we have to add the 'everybody knows' operator $\mathbf{E}$ to our language. Formulae of the form $\mathbf{E} \varphi$ are read "every agent knows that $\varphi$ ". As before, its length is $\operatorname{len}(\mathbf{E} \varphi)=1+\operatorname{len}(\varphi)$. For its semantics, we use the same models, and the satisfaction relation is as before, plus:

$$
M, w \vDash \mathbf{E} \varphi \quad \quad \text { iff } \quad \bigcup_{i \in N} R_{i}(w) \subseteq \llbracket \varphi \rrbracket_{M}
$$

The set of agents $N$ being finite, $\mathbf{E} \varphi$ is logically equivalent to $\bigwedge_{i \in N} \mathbf{K}_{i} \varphi$. Hence, multiagent EL with $\mathbf{E}$ is just as expressive as multiagent EL, and $\mathbf{E}$ could have been defined as an abbreviation in multiagent EL. According to (Lutz, 2006, Footnote 2), the addition of this operator only makes it more succinct, without increasing the computational complexity of the problem of deciding satisfiability.

However, $\mathbf{E}$ is not a master modality yet; we need yet another definition: the epistemic depth $\operatorname{ed}(\varphi)$ of a PALA formula $\varphi$ is recursively defined as follows:

$$
\begin{aligned}
\operatorname{ed}(p) & =0 \\
\operatorname{ed}(\neg \varphi) & =\operatorname{ed}(\varphi) \\
\operatorname{ed}\left(\varphi_{1} \wedge \varphi_{2}\right) & =\max \left(\operatorname{ed}\left(\varphi_{1}\right), \operatorname{ed}\left(\varphi_{2}\right)\right) \\
\operatorname{ed}\left(\mathbf{K}_{i} \varphi\right) & =1+\operatorname{ed}(\varphi) \\
\operatorname{ed}(\mathbf{E} \varphi) & =1+\operatorname{ed}(\varphi) \\
\operatorname{ed}\left(\left[! \varphi_{1}\right] \varphi_{2}\right) & =\max \left(\operatorname{ed}\left(\varphi_{1}\right), \operatorname{ed}\left(\varphi_{2}\right)\right) \\
\operatorname{ed}([\sigma] \varphi) & =\max \left(\max _{p \in \operatorname{dom}(\sigma)}(\operatorname{ed}(\sigma(p)), \varphi)\right.
\end{aligned}
$$

Let $\mathbf{E}^{k}$ stand for the string formed by operators $\mathbf{E}$ repeated $k$ times; precisely, we inductively define $\mathbf{E}^{0} \varphi=\varphi$, and $\mathbf{E}^{k+1} \varphi=\mathbf{E E}^{k} \varphi$. Now we are ready to define reduction.

$$
\operatorname{red}_{\mathrm{PALA}}(\varphi)=n_{\varphi}^{()} \wedge \mathbf{E}^{\mathrm{ed}(\varphi)}\left(\bigwedge B_{\varphi}\right)
$$

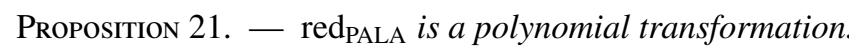

Proof. - As seen in the proof of Proposition 18, for every $n_{\chi}^{\lambda} \leftrightarrow \psi \in B_{\varphi}$ we have $\operatorname{len}\left(n_{\chi}^{\lambda} \leftrightarrow \psi\right) \leq 17+(4 \times \operatorname{len}(\varphi))$.

And as seen above in Proposition 16, $\operatorname{card}\left(B_{\varphi}\right) \leq \operatorname{len}(\varphi)$. Therefore, 
Finally, as $\operatorname{ed}(\varphi) \leq \operatorname{len}(\varphi)$, we have

$$
\operatorname{len}\left(\bigwedge B_{\varphi}\right) \leq \operatorname{len}(\varphi) \times(17+(4 \times \operatorname{len}(\varphi))+1) .
$$

$$
\begin{aligned}
\operatorname{len}\left(\mathbf{E}^{\operatorname{ed}(\varphi)}\left(\bigwedge B_{\varphi}\right)\right) & =\operatorname{ed}(\varphi)+\operatorname{len}\left(\bigwedge B_{\varphi}\right) \\
& \leq \operatorname{len}(\varphi)+\operatorname{len}(\varphi) \times(17+(4 \times \operatorname{len}(\varphi))+1)
\end{aligned}
$$

The length of $\operatorname{red}_{\mathrm{PALA}}(\varphi)$ is therefore quadratic in the length of $\varphi$.

THEOREM 22. - For every multiagent PALA formula $\varphi_{0}$, $\operatorname{red}_{\mathrm{PALA}}\left(\varphi_{0}\right)$ is a multiagent EL formula, and $\varphi_{0}$ is satisfiable if and only if $\operatorname{red}_{\mathrm{PALA}}\left(\varphi_{0}\right)=n_{\varphi_{0}}^{()} \wedge\left(\wedge_{k \leq \operatorname{ed}\left(\varphi_{0}\right)} \mathbf{E}^{k}\left(\wedge B_{\varphi_{0}}\right)\right)$ is satisfiable.

Proof. - This is essentially the same as for Theorem 19.

The problem of deciding multiagent EL satisfiability is PSPACE-complete. Therefore, we immediately get:

THEOREM 23. - The problem of deciding multiagent PALA satisfiability is PSPACE-complete.

\section{Discussion and conclusion}

We gave optimal decision procedures for the logic of public announcements and assignments PALA, adapting Lutz's abbreviation technique for PAL. We showed that the problem of deciding validity is coNP-complete for single-agent PALA, and PSPACE-complete for multiagent PALA.

Our results have also practical value because they can be directly applied to reasoning about actions in the situation calculus. Indeed, in (van Ditmarsch et al., 2007a; van Ditmarsch et al., 2011) we showed that Reiter's solution to the frame problem (Reiter, 1991; Reiter, 2001) in terms of successor state axioms can be recast in PALA: assignments allow to model 'physical' actions, and announcements allow to model epistemic observation actions. This means that one can also see our procedures as optimal decision procedures for Reiter-style reasoning about actions. In that respect, an interesting question is whether our work can be extended further to deal with so-called sensing actions. Such actions are defined in (Scherl $\&$ Levesque, 1993; Scherl \& Levesque, 2003): they are actions of the form $? \varphi$, which test whether some boolean formula $\varphi$ is true. They can be viewed as abbreviating the nondeterministic composition of two announcements: $? \varphi=! \varphi \cup ! \neg \varphi$. The problem is that the expansion of such abbreviations leads to exponential blow-up. We therefore cannot straightforwardly integrate primitive sensing actions into PALA: it is not clear how the associated reduction axiom

$$
\begin{aligned}
{[? \varphi] \mathbf{K}_{i} \psi \quad \leftrightarrow \quad } & \left(\left(\varphi \rightarrow \mathbf{K}_{i}(\varphi \rightarrow[? \varphi] \psi)\right) \wedge\right. \\
& \left.\left(\neg \varphi \rightarrow \mathbf{K}_{i}(\neg \varphi \rightarrow[? \varphi] \psi)\right)\right)
\end{aligned}
$$

could be 'compiled' into the polynomial transformation. Further evidence that the presence of sensing actions increases complexity is provided by the result in (Herzig et al., 2000) that plan verification in this case is $\Pi_{2}^{p}$-complete.

Another generalisation of our results would be to allow for non-public events, as in (Baltag et al., 1998; Bacchus et al., 1999).

\section{Acknowledgements}

Thanks to the anonymous reviewer for an impeccably precise and very useful review. 
22 Journal of Applied Non-Classical Logics. Volume Volume of the issue undefined - No. Number of the issue undefined/Year of publication undefined

Hans van Ditmarsch is also affiliated to IMSC (Institute of Mathematical Sciences Chennai), India, as a research associate.

The work of the second author was done in the framework of the French project ANR DynRes (ANR2011-BS02-011-03).

\section{References}

Bacchus, F., Halpern, J., \& Levesque, H. (1999). Reasoning about noisy sensors and effectors in the situation calculus. Artificial Intelligence, 111(1-2), 171-208.

Baltag, A., Moss, L., \& Solecki, S. (1998). The logic of common knowledge, public announcements, and private suspicions. In Proceedings of the seventh Theoretical Aspects of Rationality and Knowledge conferene (TARK) (pp. 43-46).: Morgan Kaufmann Publishers Inc.

French, T., van der Hoek, W., Iliev, P., \& Kooi, B. P. (2011). Succinctness of epistemic languages. In T. Walsh (Ed.), IJCAI 2011, Proceedings of the 22nd International Joint Conference on Artificial Intelligence (pp. 881886).: IJCAI/AAAI.

Herzig, A., Lang, J., Longin, D., \& Polacsek, T. (2000). A logic for planning under partial observability. In Proceedings of the Seventeenth Conference on Artificial Intelligence (AAAI) and the Twelfth Conference on Innovative Applications of Artificial Intelligence (IAAI) (pp. 768-773).: The AAAI Press.

Kooi, B. (2007). Expressivity and completeness for public update logic via reduction axioms. Journal of Applied Non-Classical Logics, 17(2), 231-253.

Lutz, C. (2006). Complexity and succintness of public announcement logic. In P. Stone \& G. Weiss (Eds.), Proceedings of the Fifth International Joint Conference on Autonomous Agents and Multiagent Systems (AAMAS) (pp. 137-144).

Nonnengart, A. \& Weidenbach, C. (2001). Computing small clause normal forms. In Handbook of Automated Reasoning (pp. 335-367). North Holland.

Plaza, J. (1989). Logics of public communications. In M. L. Emrich, M. Hadzikadic, M. S. Pfeifer, \& Z. W. Ras (Eds.), Proceedings of the Fourth International Symposium on Methodologies for Intelligent Systems (ISMIS) (pp. 201-216).

Reiter, R. (1991). The frame problem in the situation calculus: A simple solution (sometimes) and a completeness result for goal regression. In V. Lifschitz (Ed.), Papers in Honor of John McCarthy (pp. 359-380). Academic Press Professional Inc.

Reiter, R. (2001). Knowledge in Action: Logical Foundations for Specifying and Implementing Dynamical Systems. The MIT Press.

Scherl, R. \& Levesque, H. (1993). The frame problem and knowledge-producing actions. In Proceedings of the Eleventh National Conference on Artificial Intelligence (AAAI) (pp. 689-695).: The AAAI Press.

Scherl, R. \& Levesque, H. (2003). Knowledge, action and the frame problem. Artificial Intelligence, 144(1-2), $1-39$.

van Benthem, J., van Eijck, J., \& Kooi, B. (2006). Logics of communication and change. Information and Computation, 204(11), 1620-1662.

van Ditmarsch, H., Herzig, A., \& de Lima, T. (2007a). Optimal regression for reasoning about knowledge and actions. In Proceedings of the Twenty-Second AAAI Conference on Artificial Intelligence (pp. 1070-1075).: AAAI Press. 
van Ditmarsch, H., Herzig, A., \& de Lima, T. (2011). From situation calculus to dynamic logic. Journal of Logic and Computation, 21(2), 179-204. http://logcom.oxfordjournals.org/content/21/2/179.abstract?etoc.

van Ditmarsch, H., van der Hoek, W., \& Kooi, B. (2005). Dynamic epistemic logic with assignment. In F. Dignum, V. Dignum, S. Koenig, S. Kraus, M. Singh, \& M. Wooldridge (Eds.), Proceedings of the Fourth International Joint Conference on Autonomous Agents and Multiagent Systems (AAMAS) (pp. 141-148).: ACM.

van Ditmarsch, H., van der Hoek, W., \& Kooi, B. (2007b). Dynamic Epistemic Logic, volume 337 of Synthese Library. Springer. 